ISSN 2766-5879

\title{
Functional Hypothalamic Amenorrhoea in Adolescent girls as a Result of Stress-Related Conditions in Covid-19 Pandemic Situation
}

Anindita Jana

Consultant Gynaecologist \& Obstetrician Jhargram, WB, INDIA

\author{
*Correspondence author \\ Anindita Jana \\ Consultant Gynaecologist \& Obstetrician \\ Jhargram \\ WB \\ INDIA \\ Email: anijana2011@gmail.com
}

Submitted : 3 Aug 2021 ; Published : 20 Aug 2021

\begin{abstract}
Functional hypothalamic amenorrhoea (FHA) is a form of anovulation due to the suppression of HypothalamicPituitary-Ovarian (HPO) axis, not related to identifiable organic cause. FHA is a state of hormonal imbalance related to stress, exercising too much or consuming too few calories. In the unprecedented Covid-I9 Pandemic, there is an upsurge of FHA in adolescent girls. Being confined to 'stay at home', the phobia of gaining weight due to restricted movement is often triggering eating disorders like Anorexia Nervosa(AN);indulging in indoor overexercise, stress associated with routine change, exposure and preoccupations with social media in the changed scenario are causing a disruption of HPO axis manifesting as FHA. But FHA has serious short-term and longterm effects on the physical and mental health of the adolescent individuals. The present article aims at reviewing the causes, effects, evaluation and management of FHA in the present scenario. Adolescent girls with FHA should be carefully diagnosed and properly managed to prevent both short-term and long-term deleterious effects with appropriate and timely intervention.
\end{abstract}

Introduction

FHA is defined as hypogonadotropic hypogonadism related to an aberration of the pulsatile release of Gonadotropin releasing hormone (GnRh) from the hypothalamus [1]. The spectrum may vary from a lower mean frequency, complete absence to a fairly normal appearing LH pulsatility. In turn there is reduced oestradiol production in the ovary.

The disturbed HPO axis in FHA is typically associated with stress, weight loss, and/or excessive physical exercise. Depending on the eliciting factor, there are three classes of FHA:-

- Weight-loss related

- $\quad$ Stress-related

- Exercise-related.

The recent pandemic situation had a negative impact on adolescent physical and mental health [2]. While there has been a general tendency of weight gain due to reduced activities, it has been noted globally, there is a rise in cases of Functional hypothalamic amenorrhoea in adolescent girls related to Weight-loss, stress and overexercise in response to phobia of weight gain [3].

The tendency to overexercise, cutting down calories, causing intense weight loss is triggered by 'forced upon" routine change, lack of social connection, heightened anxiety, use of social media, according to experts. Especially, vulnerable are those with psychopathological traits similar to Anorexia Nervosa (AN). Careful history-taking and evaluation in cases of secondary amenorrhoea presenting to the Gynaecologist is important to probe into the root cause. This is of utmost importance as most young girls are 'walking well' and unaware that FHA has serious short-term and long-term hormonal, metabolic, neuropsychiatric consequences unless treatment is instituted promptly. A recently updated AAP clinical report provides guidelines for screening for eating disorders (ED), evaluating patients 'medical and psychological states for suitable management for stress-reduction and weight-gain strategies [4].

\section{Discussion}

Assessment of Hypothalamic Amenorrhoea

- Functional hypothalamic amenorrhoea (FHA) is diagnosed by exclusion of all organic causes of secondary amenorrhoea which could be disrupting the menstrual cycle.

- Laboratory tests to check hormonal status including Thyroid hormone, Prolactin and Estrogen is indicated in those with persistent amenorrhoea of 3 months or more or interval more than 45 days.

- Progesterone challenge causes no withdrawal bleed in cases of FHA.

- Evaluating causal and concomitant conditions is of paramount importance. 
Mechanism of suppression of HPO axis leading to FHA Figure.1 discusses the mechanism of suppression of HPO axis leading to FHA [5]. The process by which GnRh is suppressed is multi-factorial, as there are several neuromodulatory signals that alter hypothalamic GnRh function, both inhibitory and stimulatory. GnRH may be suppressed by decreased Insulinlike growth factor (IGF-1), increased cortisol, increased ghrelin, decreased triiodothyronine (T3) and reduced leptin. Kisspeptin signalling has been implicated as the common intermediate signalling factor, acting downstream of leptin \& other neuromodulatory signalling systems to regulate $\mathrm{GnRH}$ secretion. Lack of GnRH decreases the mean frequency of LH pulsatility and FSH synthesis. This, in turn, causes decreased oestradiol synthesis by the ovaries which leads to amenorrhoea. (FHA).

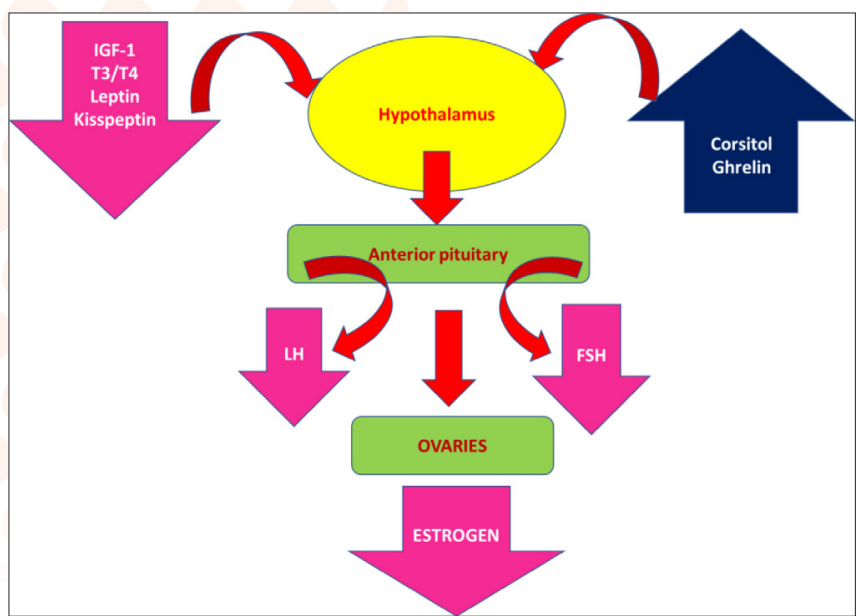

Figure 1: The effect of neuroendocrine signalling factors associated with FHA causing suppression of HPO axis [5]

\section{Effects of Hypoestrogenism}

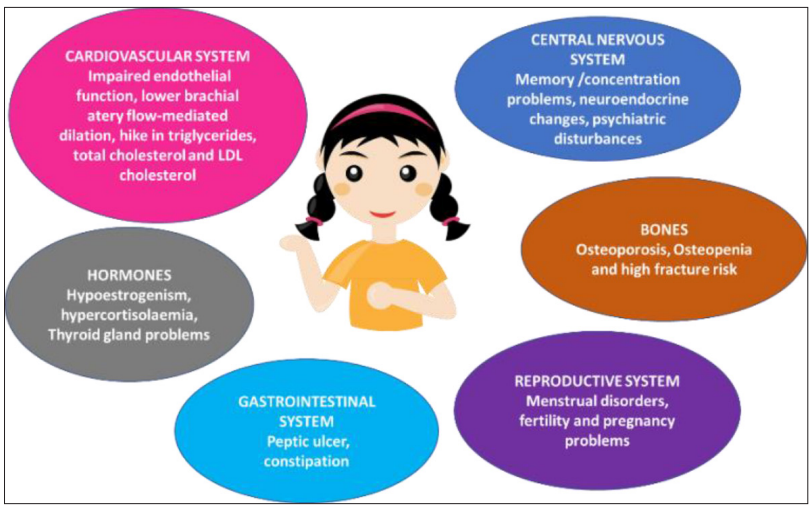

Figure 2: Consequences of Hypoestrogenism in FHA [6].

Hypoestrogenism has profound short-term and long-term consequences in adolescent girls. They have a greater risk of developing complications in the reproductive, cardiovascular, skeletal and neuroendocrine systems. Psychiatric comorbidities including anxiety and mood disorders, low self-esteem, inability to cope with stress and depression are attributed to hypoestrogenism $[6,7]$.
Estrogen deficiency is a risk factor for osteoporosis. Girls with exercise-induced FHA, and particularly those with lower body weights and restrictive food habits have impaired bone growth and lower BMD Z-scores in the lumbar spines in adulthood compared to their normal menstruating counterparts [8].

\section{Management}

- Treatment should be initiated as soon as possible. The major problem is resistance and denial on the part of the adolescent patients. It is recommended that clinicians evaluate patients for in-patient treatment who have FHA with severe bradycardia, hypotension, orthostasis, and/or electrolyte imbalance $[9,10]$. Special attention is required to prevent relapse by proper aftercare after recovery.

- The mainstay of treatment is correction of energy imbalance to restore the HPO axis. This requires increased calorie consumption, and/or improved nutrition and/ or decreased exercise activities. The primary goal is weight gain.

- Psychological support is essential for adolescent girls with cognitive behaviour therapy (CBT) [2,9].

- Oral Contraceptive pills (OCP)s are often prescribed to induce cyclic bleeding. However recent studies suggest that OCPs are of no value for the sole purpose of regaining menses or reversing bone loss,rather they may create a false sense of well-being and remove the motivation for weight-gain.[9].One study suggested that increasing energy intake to $>30 \mathrm{kcal} / \mathrm{kg}$ of fat-free mass per day may reverse the amenorrhoea; in another trial, the recovery of menstrual function is predicted by the initiation and maintenance of weight-gain targeted between the $15 \%$ to $20 \%$ of BMI within 12 months, but more data is required to confirm this finding [10,11]. Notably, some girls do not spontaneously undergo resumption of menses even after weight gain emphasizing on the fact that underlying psychological factors need to be addressed as well.

- Short-term transdermal Estrogen therapy with cyclic oral progestin is advisable in adolescent girls who have not had return of menses after a reasonable trial of nutritional, psychological and/or modified exercise intervention [12].

- Available data do not support, rather suggest against the use of bisphosphonates, testosterone, denosumab, and leptin to improve BMD in adolescent girls with FHA [13]. Weight restoration, Calcium and Vitamin D supplements and the resumption of spontaneous menses is the mainstay of treatment.

- Exogenous leptin administration restored ovulatory cycles in 3 of 8 women of normal weight who had hypothalamic amenorrhoea [14]. However recombinant human leptin led to $4.3 \%$ decrease in weight in 5 individuals who completed the 3 -month trial, which limits the potentiality of leptin to be used as a therapy in women who are already experiencing weight loss as in AN.

- The use of GnRH pumps, Kisspeptin (for initiating secretion of $\mathrm{GnRH}$ ) has been studied in small pilot samples [15]. However, more research is needed to demonstrate LH pulsatility.

- Cognitive Behavioural Therapy (CBT) is a successful treatment option for FHA due to psychological stress 
and results in resumption of ovarian activity. Success for CBT underscores the need in FHA girls to alter patterns of thinking and behaviour as well as an option of nonmedication alternative form of treatment [16].

\section{Conclusion}

FHA is a reversible disorder caused by stress related to weight loss, excessive exercise/or traumatic mental experience. Treatment of FHA should be started as soon as possible, and it should primarily involve lifestyle modification. It may be supplemented by pharmacological treatment to allay stress and anxiety. Importantly, treatment is not often long-term, and it results in recovery of majority of adolescent patients. Effective therapy, based on multidisciplinary approach, can protect patients from negative impact on fertility, cardiovascular system and bone health, as well as reducing mental morbidity. In this current scenario of ongoing pandemic, we need to highlight upon this issue and counsel children, adolescents, parents, teachers to safeguard physical and mental health. Multidisciplinary team including the Primary Physician, Gynaecologist, Endocrinologist, Psychiatrist, Nutritionist and Dietician should be approached to improve the outcome in this setting of new-normal age.

The goal of the future is to introduce new drugs that prove to be more effective than HRT and satisfactorily re-establish the $\mathrm{HPO}$ axis in its original tune.

\section{Funding}

The research received no external funding

\section{Conflict of interest}

The author declares no conflict of interest

\section{References}

1. Gordon CM(2010) Functional hypothalamic amenorrhoea. N Engl J Med 363: 365-371.

2. Phelan N, Behan AL, Owens L (2021) The impact of the Covid-I9 Pandemic on Women's reproductive health. Front. Endocrinol 12: 642755.

3. Giles DE, Berga SL (1993) Cognitive and psychiatric correlates of Functional hypothalamic amenorrhoea:a controlled comparison. Fertility Steril 60: 486-492.

4. Hornberger LL, Lane MA and THE COMMITTEE ON ADOLESCENCE (2021) Identification and Management of Eating Disorders in Children and Adolescents, Pediatrics 147: e2020040279

5. Podfigurna A, Meczekalski B (2021) Functional hypothalamic amenorrhoea: A stress-based disease Endocrines 2: 203-211.

6. Shufelt CL, Torbati T, Dutra E (2017) Hypothalamic Amenorrhea and the Long-Term Health Consequences. Semin Reprod. Med 35: 256-262.

7. Miller KK, Klibanski A (1999) Amenorrheic bone loss. Journal Clinical Endocrinol Metabol 84: 1775-1783.

8. Meule A, Schrambke D, Furst Loredo A, Schlegl S, Naab $S$, et al (2021) Inpatient treatment of anorexia nervosa in adolescents: A 1-year follow-up study. Eur. Eat. Disorders
Rev 29: 165-177.

9. Michopoulos V, Mancini F, Loucks TL, Berga SL (2013) Neuroendocrine recovery initiated by Cognitive Behavioural Therapy in women with functional hypothalamic amenorrhoea: a randomized controlled trial. Fertil. Steril 99: 2084-2091.

10. Bergström, I, Crisby M, Engström AM, Hölcke, M Fored M, et al. (2013) Women with anorexia nervosa should not be treated with estrogen or birth control pills in a bonesparing effect. Acta Obstet. Gynecol. Scand 92: 877-880.

11. Roberts RE, Farahani L, Webber L, Jayasena C (2020) Current understanding of hypothalamic amenorrhoea. Ther. Adv. Endocrinol. Metab 11: 2042018820945854.

12. Gordon CM, Ackerman KE, Berga SL, Kaplan JR, Mastorakos G, et al. (2017) Functional Hypothalamic Amenorrhea: An Endocrine Society Clinical Practice Guideline, J Clin Endocrinol Metab 102: 1413-1439

13. Golden NH (2003 Osteopenia and osteoporosis in anorexia nervosa. Adolesc. Med 14: 97-108.

14. Grinspoon S, Gulick T, Askari H, Landt M, Lee K, et al. Serum leptin levels in women with anorexia nervosa. $J$ Clin Endocrinol Metab 81: 3861-3863.

15. Jayasena CN, Abbara A, Veldhuis JD, Comninos AN, Ratnasabapathy R, et al. (2014) Increasing LH pulsatility in women with hypothalamic amenorrhoea using intravenous infusion of Kisspeptin-54. J Clin Endocrinol Metab 99: E953-961.

16. Berga SL, Marcus MD, Loucks TL, Hlastala S, Ringham $\mathrm{R}$, et al. (2003) Recovery of ovarian activity in women with functional hypothalamic amenorrhea who were treated with cognitive behavior therapy. Fertil. Steril 80: 976-981.

Copyright: (C2021 Anindita Jana. This is an open-access article distributed under the terms of the Creative Commons Attribution License, which permits unrestricted use, distribution, and reproduction in anymedium, provided the original author and source are credited. 